\title{
Beam me up, Spotty: Toward a new understanding of the physics of massive star photospheres
}

\author{
Alexandre David-Uraz ${ }^{1}$, Gregg Wade $^{2}$ and Stan Owocki ${ }^{3}$ \\ ${ }^{1}$ Queen's University, Canada \\ email: adavid-uraz@astro.queensu.ca \\ ${ }^{2}$ Royal Military College, Canada \\ ${ }^{3}$ University of Delaware, USA
}

\begin{abstract}
For 30 years, cyclical wind variability in $\mathrm{OB}$ stars has puzzled the astronomical community. Phenomenological models involving co-rotating bright spots provide a potential explanation for the observed variations, but the underlying physics remains unknown. We present recent results from hydrodynamical simulations constraining bright spot properties and compare them to what can be inferred from space-based photometry. We also explore the possibility that these spots are caused by magnetic fields and discuss the detectability of such fields.
\end{abstract}

Keywords. Hydrodynamics, methods: numerical, stars: winds, outflows

\section{Introduction}

Massive stars exhibit cyclical spectral variability on timescales of hours to days. One seemingly ubiquitous manifestation of these variations is the presence of so-called "discrete absorption components" (DACs, Howarth \& Prinja 1989): additional absorption features within the P Cygni absorption troughs of wind-sensitive UV resonance lines which progress from low to near-terminal velocity. They are believed to be rotationally modulated. The generally-accepted hypothesis which explains their formation is the "corotating interaction regions" model (CIR, Mullan 1986). Bright spots on the surface of the star locally drive an enhanced wind, leading to spiral-like structures (Cranmer \& Owocki 1996, henceforth referred to as CO96). The most important feature of this model is the presence of "velocity kinks" in the wind; the driving of extra material by the bright spots cannot be sustained (the wind is overloaded) and that material eventually decelerates, which results in a velocity plateau, thus leading to an accumulation of matter at a given velocity which causes the extra absorption associated with DACs.

\section{Modern constraints and revisited model}

CO96 provide a phenomenological explanation for the DAC phenomenon. Consequently, the presence of bright spots was not a priori physically motivated (nor constrained). However, with the advent of space-based photometry (e.g. the MOST space telescope), the precisely-determined amplitude of light-curve variations places very strong constraints on the size and contrast of putative spots on the surface of the star. Ramiaramanantsoa et al. (2014) find a 10 mmag amplitude for $\xi$ Per, constraining any spots present to be much more modest than those used by CO96. It is unclear whether velocity kinks can be formed with such modest spots. One possible way to facilitate kink formation is by including ionization effects, which were not taken into account by CO96. 

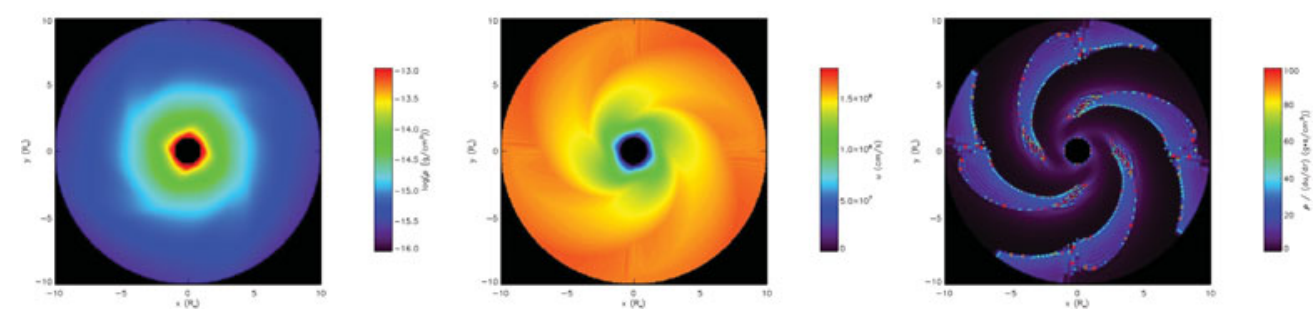

Figure 1. Preliminary 2D hydrodynamic models show that four spots with $10^{\circ}$ radius and $33 \%$ brightness enhancement allow us to qualitatively reproduce the conditions necessary to obtain DACs. From left to right: density profile, radial velocity profile, density divided by the radial velocity gradient (this proxy variable acts like the optical depth and the bright trailing edges indicate the presence of velocity kinks).

Following up on that work, we aim to study the effects of adding the ionization correction $(\delta)$ in hydrodynamic wind simulations, using the $\mathrm{VH}-1$ package. 1D simulations show that for high values of the $\delta$ parameter (Abbott 1982), the wind becomes overloaded and we obtain a coasting solution, even without spots. While this work is quite preliminary, 2D hydrodynamical simulations based on realistic stellar parameters (in this case, $\xi$ Per, with $10^{\circ}$ spots and a $33 \%$ brightness enhancement) and including additional wind physics (ionization effect, finite disk correction) show that we can reproduce CIRs and even obtain the desired velocity kinks (see Fig. 1).

\section{The role of magnetic fields}

Magnetic fields constitute a popular hypothesis to explain the surface nonuniformities apparently needed to form CIRs. While only a small fraction of OB stars are known to host detectable fields (about 7\%, Wade et al. 2013), most known magnetic massive stars have a large scale, essentially dipolar field. However, David-Uraz et al. (2014) effectively rule out large-scale magnetic fields as the physical cause of DACs. Recent models (e.g. Cantiello et al. 2009) suggest the existence of a sub-surface convection layer in massive stars which could produce small-scale surface magnetic fields. Kochukhov \& Sudnik (2013) have characterized the detectability of randomly distributed magnetic spots. For $v \sin i<50 \mathrm{~km} \mathrm{~s}^{-1}$, one would expect to detect $10^{\circ}$ magnetic spots with a field strength of over $100 \mathrm{G}$ (with a 0.5 filling factor); to produce a $10 \mathrm{mmag}$ photometric variation, such a spot should have a field strength of about $330 \mathrm{G}$ (using the formula of David-Uraz et al. 2014), which should therefore be detectable with current instrumentation. Further work on both the numerical and observational fronts will be required to better understand and constrain the role of small-scale magnetic fields in producing DACs.

\section{References}

Abbott, D. C. 1982, ApJ 259, 282

Cantiello, M., Langer, N., Brott, I., et al. 2009, A\& A 499, 279

Cranmer, S. R. \& Owocki, S. P. 1996, ApJ 462, 469

David-Uraz, A., Wade, G. A., Petit, V., et al. 2014, ArXiv e-prints

Howarth, I. D. \& Prinja, R. K. 1989, ApJS 69, 527

Kochukhov, O. \& Sudnik, N. 2013, A\&A 554, A93

Mullan, D. J. 1986, A\&\&A 165, 157

Ramiaramanantsoa, T., Moffat, A. F. J., Chené, A.-N., et al. 2014, MNRAS 441, 910

Wade, G. A., Grunhut, J., Alecian, E., et al. 2013, ArXiv e-prints 